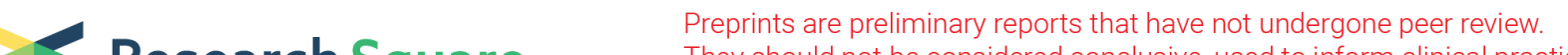 Research Square They should not be considered conclusive, used to inform clinical practice, or referenced by the media as validated information.
}

\section{Evaluation of Standard Dosing for Selected Broad Spectrum Hydrophilic Antibiotics in Critically III Patients with Augmented Renal Clearance: An Observational Study}

\author{
Majed Almutairi \\ King Abdulaziz Medical City \\ Khalid Al Sulaiman ( $\square$ alsulaimankh@hotmail.com ) \\ King Abdulaziz Medical City \\ Sultan Alenazi \\ King Saud bin Abdulaziz University for Health Sciences \\ Ramesh Vishwakarma \\ King Abdullah International Medical Research Center \\ Ohoud Aljuhani \\ King Abdulaziz University
}

\section{Research Article}

Keywords: Augmented renal clearance (ARC), Critically ill, Confirmed infections, Broad spectrum hydrophilic antibiotics (Meropenem, Imipinem, Piperacillin/Tazobactam), The eradication of pathogen at 10-14 days, The resistance of pathogen after three days, Persistence of pathogen after 3 days, 30-daysICU mortality, ICU Length of Stay (days), Hospital length of stay (days).

Posted Date: July 27th, 2021

DOI: https://doi.org/10.21203/rs.3.rs-726484/v1

License: (c) (1) This work is licensed under a Creative Commons Attribution 4.0 International License. Read Full License 


\section{Abstract}

Background: Inappropriate antibiotics dosing in critically ill patients with augmented renal clearance (ARC) may be associated with pathogens resistance and worse outcomes. Unfortunately, studies regarding the relationship between $A R C$ and clinical outcomes in patients treated with antibiotics medication are rare. The study aims to evaluate the efficacy and clinical outcomes of selected broad spectrum hydrophilic antibiotics (Meropenem, Imipinem, Piperacillin/Tazobactam) in augmented renal function critically ill patients with confirmed infections.

Methods: A retrospective cohort study in critically ill patients who were admitted to intensive care units (ICUs) at King Abdulaziz Medical City (KAMC)-Riyadh and received broad-spectrum Hydrophilic antibiotics (Meropenem, Imipenem, or Piperacillin/Tazobactam) with confirmed infection. All the patients who met our inclusion criteria during the study period (01/01/2018 - 31/12/2019) were included. Eligible patients have been divided into two groups (augmented renal clearance (ARC) Vs. Non-ARC) according to the estimated creatinine clearance $(\mathrm{CrCl})$ to assess pathogen eradication at 10-14 days.

Results: A total of 2682 patients were screened; 133 patients were included in the study. The eradication of pathogen at 10-14 days in ARC group was non-inferior to non-ARC group (OR 1.08; 95\% Cl, 0.41-2.78 p $=0.88$ ). The Resistance (OR $0.78 ; 95 \% \mathrm{Cl}, 0.25-2.40 \mathrm{p}=0.66$ ), and Persistence after 3 days (OR 0.88; $95 \%$ $\mathrm{Cl}, 0.35-2.18 \mathrm{p}=0.78$ ) were not significantly different between the groups. In other words, patients with ARC have the same rate of resistance and persistence of pathogen after three days. There was no difference in the 30-day mortality between the two groups (OR $0.22 ; 95 \% \mathrm{Cl}, 0.04-1.40 \mathrm{p}=0.11$ ). Also, there was no difference in the ICU length of stay (LOS) nor in hospital LOS between the two groups (22.0 days vs. 17.5 days, $p=0.37$ ), and ( 51.0 days vs. 30.0 days, $p=0.07$ ) respectively.

Conclusion: Using standard dosing of hydrophilic broad-spectrum antibiotics in ARC patients was not inferior to non-ARC patients in terms of the pathogen eradication, resistance, and persistence. Further randomized clinical and interventional studies are required to confirm our findings.

\section{Background}

Over the past decade, researchers began to understand and describe a phenomenon manifested by markedly increasing the renal clearance, known as augmented renal clearance (ARC) (1). ARC is defined by Creatinine Clearance $(\mathrm{CrCl})$ values ranging from more than $120 \mathrm{ml} / \mathrm{min}$ to more than $150 \mathrm{ml} / \mathrm{min}$ using the Cockcroft-Gault formula $(2,3)$. ARC estimated to be $20-65 \%$ in critically ill patients with a wide range of risk factors, such as patients younger than 50 years old, male gender, had a recent history of trauma, subarachnoid hemorrhage, burns, or had lower critical illness severity scores (e.g., sequential organ failure assessment score (SOFA), Acute Physiology and Chronic Health Evaluation (APACHE II) (4, 5). This phenomenon might lead to an increase in the rate of renally eliminated medications (1). Therefore, standard doses of some antimicrobials may not be sufficient, and a higher dose needed in selected patients with $\operatorname{ARC}(7,8,9)$. 
Many of the studies published about ARC were focused on the antimicrobial therapy because it might lead to the emergence of drug resistance (6). Beta-lactams antibiotics are a time-dependent antibacterial activity that will be highly eliminated by the kidney and decrease the exposure time of the drug. Several studies in this field have reported beta-lactam treatment failures based on sub-therapeutic serum level attainment when using standard dosing regimens in patients with $\operatorname{ARC}(6,10)$. A Retrospective observational study conducted by Drust et al. 2011 in ICU patients with $\mathrm{CrCl}$ greater than $120 \mathrm{~mL} / \mathrm{min}$ by measuring meropenem plasma concentrations found that $67 \%$ of patients required higher doses of meropenem than standard doses to achieve therapeutic plasma concentration (11). Moreover, Carlier et al. 2013, conducted a prospective observational study in the ICU population, found that high creatinine clearance is an independent predictor for not achieving target concentrations levels of meropenem and piperacillin/tazobactam (12). Another prospective observational study conducted by Huttner et al. 2015 in ICU patients with ARC found a correlation with ARC and undetectable antimicrobials concentrations without significant clinical failure (13).

Inappropriate antibiotics dosing in critically ill patients with ARC may be associated with pathogens resistance as well as worse outcomes. Unfortunately, studies regard the relationship between ARC and clinical outcomes in patients treated with antibiotics medication are scarce. Our study will evaluate the efficacy as well the clinical outcomes of selected broad spectrum hydrophilic antibiotics (Meropenem, Imipinem, Piperacillin/Tazobactam) in augmented renal function critically ill patients with confirmed infections.

\section{Methods}

\section{Study design}

A retrospective cohort study in critically ill patients who received standard dosing of selected broadspectrum Hydrophilic antibiotics (Meropenem, Imipenem, or Piperacillin/Tazobactam) for confirmed infection (Bacteremia, pneumonia, and Urinary Tract Infection (UTI)). The diagnosis of infection was confirmed by lab culture depend on the site of infection. All the patients who met our inclusion criteria during the study period (01/01/2018 - 31/12/2019) were included. Eligible patients have been divided into two groups (augmented renal clearance (ARC) Vs. Non-ARC) according to the estimated creatinine clearance ( $\mathrm{CrCl}$ ) (By Cockroft equation). Patients were followed during their ICU Length of stay (LOS) until ICU discharge after improving or in-hospital death, whichever occurred first.

\section{Participants}

Patients were enrolled in the study if they were critically ill admitted to ICU, aged 18-65 years, had a confirmed infection (Bacteremia, pneumonia, and Urinary Tract Infection (UTI)) and received standard dosing of broad-spectrum Hydrophilic antibiotics (Meropenem, Imipenem or Piperacillin/Tazobactam) for these infections. Patients were excluded if the Weight $<40 \mathrm{~kg}$ or BMl $<16 \mathrm{Kg} / \mathrm{m} 2$, on any dialysis, no-code status, previous multi-drug resistance within three months of admission, if the patient received any antibiotics during 90 days before admission or previous admission within 90 days if the first culture for 
the defined infection resistance to selected antibiotics, an inappropriate dose of antibiotics for selected indication, early dose adjustment (less than three days), duration of antibiotics less than three days, uncontrolled source of infection.

\section{Setting}

This study was conducted in the adult medical, surgical, trauma, and burn ICUs at KAMC, a tertiary-care academic referral hospital in Riyadh, Saudi Arabia. The ICU admits medical, surgical, trauma, burn patients and operates as closed units with 71 ICU beds capacity with $24 / 7$ onsite coverage by critical care board-certified intensivists (14).

\section{Ethics approval and consent to participate}

The study was approved by the Ministry of National Guard Health Affairs Institutional Review Board, Riyadh, Saudi Arabia (Study Number: RC20/012/R). Participants' confidentiality was strictly observed throughout the study using the anonymous unique serial number for each subject and restricting data only to the investigators. Informed consent was not required due to the research's method as per the governmental and local research center's policy.

\section{Data collection}

We collected the following information, demographic data (Table 1, Supplemental), Acute Physiology And Chronic Health Evaluation II (APACHE II) and Sequential Organ Failure Assessment (SOFA) scores, comorbidities, vital signs, laboratory tests within 24 hours of ICU admission, and $\mathrm{CrCl}$ (By Cockroft equation) at day \#1 AND \#3, Antibiotics, baseline cultures, follow-up cultures, evaluation of pathogen resistance, persistence after three days and eradication at 10-14 days. Additionally, ICU LOS, hospital LOS, and ICU mortality were collected and followed. Patients were followed during ICU LOS until ICU discharge after improving, or in-hospital death, whichever occurred first.

\section{Outcomes}

The aim of this study is to evaluate the efficacy and clinical outcomes of using standard dosing of selected broad spectrum antibiotics. The primary endpoint was to assess the eradication of pathogen at 10-14 days. The secondary endpoints include the development of resistance and persistence pathogen growth after three days, ICU mortality within 30-day, ICU LOS, and hospital LOS.

\section{Definition (s).}

The ARC defined as an estimated $\mathrm{CrCl} \geq 130 \mathrm{ml} / \mathrm{min}$ and Non-ARC as an estimated $\mathrm{CrCl}$ 50-129 ml/min.

a. Estimated $\mathrm{CrCl}$ by Cockcroft-Gault (CG) method. $\mathrm{CrCl}($ male $)=([140$-age $($ years $)] \times$ weight $($ in $\mathrm{kg})] /(\mathrm{SrCr}(\mathrm{mg} / \mathrm{dL}) \times 72)$ $\mathrm{CrCl}($ female $)=\mathrm{CrCl}($ male $) \times 0.85$ 
b. The average of estimated $\mathrm{CrCl}$ depend on day 0 and day 3 from admission. If Body Mass Index $(\mathrm{BMI})>30$ will use Adjusted Body weight (adjBW) and Adjusted Creatinine Clearance (AdjCrCl), BMI<30 will use Actual Body weight (ActBW) and actual CrCl.

c. Infection was identified through the blood, urine, and/or respiratory cultures. The bacterial growth considered significant if the growth is $\geq$ of $100,000 \mathrm{CFU} / \mathrm{ml}$ in sputum or endotracheal aspiration shows; bronchoalveolar lavage (BAL) shows growth $\geq 0$ of $10,000 \mathrm{CFU}$ of single organism/ml for protected specimen brushes (PSBs), and $\geq 100,000 \mathrm{CFU}$ of single organism/ $\mathrm{ml}$ for BAL fluid. Additionally, urinary cultures were considered significant if showing a growth of $\geq 100,000 \mathrm{CFU} / \mathrm{ml}$ of no more than two species of microorganisms. Cultures were excluded if the laboratory reported them as a "contaminant sample" (15).

d. Resistant organisms were defined as the non-susceptibility to at least three or more antimicrobial agents. Susceptibility of microorganisms created using documents and breakpoints based on Clinical Laboratory Standards Institute (CLSI) (16).

e. Persistence organisms were defined as the presence of the same micro-organism in the repeated culture after three days (17).

f. Standard dosing of antibiotics in Lexicomp ${ }^{\circledR}$ depend on micro-organism and type of infection:

\section{Meropenem:}

Respiratory, Bacteremia and UTI infections: 1 gram every 8 hours.

II. Imipenem:

Respiratory, Bacteremia and UTI infection: 500 mg every 6 hours.

III. Piperacillin/Tazobactam:

1. Respiratory infection: 4.5 gram every 6 hours.

2. Bacteremia infection: 4.5 gram every 6 hours.

3. UTI infection: 3.375 gram every 6 hours, if pseudomonas 4.5 gram every 6hours.

\section{Data management and Statistical analysis}

We summarized categorical variables as number (percentage) and numerical variables (continuous variables) as mean and standard deviation (SD). The normality assumptions were assessed for all numerical variables using statistical test (i.e. Shapiro-Wilk test) and using graphical representation (i.e. histograms and Q-Q plots). We compared categorical variables using the chi square or Fisher exact test, normally distributed numerical variables with the t test, and other quantitative variables with the MannWhitney $U$ test. Baseline characteristics, baseline severity and outcome variables were compared between the two groups. No imputation was made for missing data as the cohort of patients in our study was not derived from random selection.

Multivariate logistic and generalized linear regression analyses were used to determine the relationship between the two groups and the different outcomes considered in this study. We considered a P value of 
$<0.05$ statistically significant and used SAS version 9.4 for all statistical analyses.

\section{Results}

\section{Demographic and Clinical Characteristics}

Among 2682 critically ill patients screened, 133 patients were included in the study who received the standard dosing of selected antibiotics at ICU. Patient characteristics and demographic data are described in (Table 1, Supplemental). Patients were divided to two groups based on renal function (ARC group $=66$ patients vs non-ARC group $=67$ patients). Of those included in ARC vs non-ARC, $36(54.5 \%)$ vs 32 (47.8\%) patients received Piperacillin/Tazobactam, 25 (37.9\%) vs 27 (40.3\%) patients received Meropenem, and $5(7.6 \%)$ vs $8(11.9 \%)$ patients received Imipenem. The distribution of infections and types of pathogens between the groups were the same and there are no differences. (Table 1)

Most of the patients were men (83.5\%), and the mean age of the patients was $42.2 \pm 14.7$ years. The mean $\mathrm{CrCl}$ depending on BMI during ICU Day admission and day 3 are $161.7(27.22 \%)$ and 97.1 (19.44\%) for the first and second group respectively (Table 1, Supplemental). The most common comorbidities were diabetes mellitus (17.3\%), and hypertension (23.3\%); comorbidities were significantly different between the two groups (Table 2, Supplemental).

There were notable differences between the two groups. Patients who in ARC group were traumatic, neuro and burn patients $34(51.5 \%), 11(16.7 \%) 9(13.6 \%)$ respectively, $p=0.0003)$. The baseline severity scores (i.e., APACHE II and SOFA), Glasgow coma score (GCS), lactic acid, platelets count, and PaO2/FiO2 ratio within 24 hours of ICU admission, were not significantly different between the two groups. The median APACHE II score was 15 , while the median SOFA score was 7. The $87.2 \%$ of patients needed MV within 24 hours of ICU admission. (Table 1, Supplemental).

\section{Primary endpoint}

The eradication of pathogen at 10-14 days in ARC group was non-inferior to non-ARC group $72.7 \%$ vs $71.6 \%, p=0.09$. Moreover, after adjusting for the patient's gender and mean weight used depending on BMI during ICU stay, eradication of pathogen at 10-14 days (OR 1.08; $95 \% \mathrm{Cl}, 0.41-2.78 \mathrm{p}=0.88$ ) were not significantly different between the groups (Table 2).

\section{Secondary endpoints}

The resistance after three days was $18.2 \%$ vs $16.4 \%, \mathrm{P}=0.79$ and persistence was $31.8 \%$ vs $31.3 \%, \mathrm{p}=$ 0.95 respectively. Moreover, after adjusting for the patient's gender and mean weight used depending on BMI during ICU stay, the resistance (OR $0.78 ; 95 \% \mathrm{Cl}, 0.25-2.40 \mathrm{p}=0.66)$, and persistence after three days (OR $0.88 ; 95 \% \mathrm{Cl}, 0.35-2.18 \mathrm{p}=0.78$ ) were not significantly different between the groups. In other words, patients with ARC who received standard dosing have the same rate of resistance and persistence of pathogen after three days (Table 2). 
The 30 day-mortality was $4.5 \%(n=3)$ vs $13.4 \%(n=9), P=0.07$ in ARC and non-ARC groups respectively. Moreover, after adjusting for the patient's Gender and mean weight used depending on BMI (OR 0.22; $95 \%$ $\mathrm{Cl}, 0.04-1.40 \mathrm{p}=0.11$ ) were not significantly different between the groups (Table 2). Additionally, no differences in ICU LOS (22.0 days vs. 17.5 days, $p=0.37$ ), and hospital length of stay ( 51.0 days vs. 30.0 days, $p=0.07$ ) (Table 2$)$.

\section{Discussion}

Our study is a single-center retrospective cohort of critically ill patients admitted to different ICUs with confirmed infections. It aims to evaluate the efficacy and the association between augmented renal function (ARC) and various clinical outcomes of selected broad spectrum hydrophilic antibiotics (Meropenem, Imipenem, Piperacillin/Tazobactam). Patients with ARC were younger and mainly admitted with a traumatic injury which is a common risk factor for ARC $(4,5)$. In our cohort, there was no significant difference in terms of infection distribution and the types of pathogens. However, there was a significant difference in terms of the prescribed beta-lactam antibiotics between both groups;

Piperacillin/Tazobactam was the most administered antibiotics (ARC vs. non-ARC, 36 (54.5\%) vs. 32 $(47.8 \%))(13)$.

The main finding of eradicating pathogen at 10-14 days by utilizing standard dosing of broad-spectrum hydrophilic antibiotics in the ARC group was non-inferior to the non-ARC group with a $p$-value of $(p=0.88)$ after adjusting for possible confounders. Several studies have addressed the link between ARC treatment failure based on antibiotics serum level measurements without assessing pathogen eradication $(6,7,8,9,10)$. In contrast, in our cohort we identified the treatment failure based on various clinical factors such as the eradication and the persistence of the pathogen. Our finding agrees with the results of one retrospective cohort study, where patients with ARC have a subthreshold beta-lactam antibiotics concentration that was not associated with clinical failure. However, this study was limited by MIC testing, patient heterogeneity, and an estimated $\mathrm{Crcl}$ calculation instead of measurement (13).

Additionally, we assessed the resistance after three days of using standard antibiotics dosing and found no significant difference among the groups with a $p$-value of $(p=0.66)$. Clinical failure could be identified by the persistence and resistance of the pathogen after three days, which all were not statistically different in our cohort. In contrary, a prospective observational study found that $27 \%$ of patients with ARC $(\mathrm{CrCl}>130 \mathrm{~mL} / \mathrm{min} / 1.73 \mathrm{~m} 2)$ had a therapeutic failure more often than non-ARC patients $(13 \%)(\mathrm{p}=$ 0.04) (18). However, the external validity of our outcome could be limited due to the small sample size in our study.

ARC group was similar in infection persistence rate after three days compared to non-ARC $(p=0.78)$. The association between ARC and infection persistence rate was not clearly assessed in previous studies. A single-center prospective study was conducted in the Surgical and Trauma ICU to assess the rate of therapeutic failure by evaluating the impaired clinical response, which is defined as persistent or recurrent clinical and biological symptoms of the initial infection. This study showed that within the first three days 
of antibiotics therapy, a mean $\mathrm{CrCl}$ value $\geq$ of $170 \mathrm{~mL} / \mathrm{min}$ was not associated with low antibiotic exposure and did not lead to therapeutic failure (19). Similarly, we defined treatment failure by infection persistence after three days and found no significant difference between the two groups. The negative impact of $A R C$ related to decreasing the antibiotic concentration and its correlation with infection persistence needs to be further investigated in critically ill patients.

There is an increasing concern about the therapeutic failure due to ARC and its association with prolonged ICU length of stay and mortality. We have investigated the effect of ARC on ICU-related outcomes and found no significant differences in the ICU mortality rate as well as ICU length of stay. Our finding agrees with the results of a sub-study driven from a large clinical trial that assessed ARC's influence determined by creatinine clearance measurement on the ICU-related clinical outcomes. Similarly, this study has found no significant difference in ICU-free days and 90-day mortality with $p$ values of 0.89 and 0.33 , respectively (20-21). The presumed ARC negative impact on clinical outcomes due to lower antibiotic exposure was not evident in our study and other studies (120). This point could be justified that in ARC patients, the low antibiotics exposure is not only the main driving factor for negative outcomes but also other factors such as the severity of illness and organ dysfunction.

Our study might have strength points to address, such as the clear definition of ARC and the specific inclusion criteria and our adjustment for possible confounders and including several types of ICU patients. However, our conclusion might have several limitations. First, the study's retrospective nature, data extracted from a single-center, and the small sample size could limit the study's external validity. Second, the exact MIC value was not reported, and the unavailability of antibiotics serum concentration measurements. Lastly, we utilized an estimated $\mathrm{CrCl}$ by Cockcroft-Gault method due to the lack of $24 \mathrm{hrs}$ $\mathrm{CrCl}$ measurements, to overcome the estimated $\mathrm{CrCl}$ shortcomings, we calculated the average of estimated $\mathrm{CrCl}$ depend on day zero and three from admission taking into consideration the BMI.

\section{Conclusion}

Using standard dosing of hydrophilic broad-spectrum antibiotics in ARC patients was not inferior to nonARC patients in terms of pathogen eradication, resistance, and persistence. Further randomized clinical and interventional studies are required to confirm our findings.

\section{Abbreviations}

King Abdulaziz Medical City (KAMC) - Ministry of National Guard Health Affairs (MNGHA), Augmented renal clearance (ARC), intensive care units (ICUs), Creatinine Clearance ( $\mathrm{CrCl})$, Cockcroft-Gault equation (CG), Body Mass Index (BMI), sequential organ failure assessment score (SOFA), Acute Physiology and Chronic Health Evaluation (APACHE II), Urinary Tract Infection (UTI), Length of stay (LOS), Adjusted Body weight (adjBW), Adjusted Creatinine Clearance (AdjCrCl), Actual Body weight (ActBW), bronchoalveolar lavage (BAL), protected specimen brushes (PSBs), Clinical Laboratory Standards Institute (CLSI), standard deviation (SD), Glasgow coma score (GCS) 


\section{Declarations}

\section{Acknowledgements}

Not applicable.

\section{Authors' contributions}

All authors contributed to data collections, analysis, drafted, revised, and approved the final version of the manuscript.

\section{Funding}

None.

\section{Ethical Approval and consent to participate}

The study was approved by the Ministry of National Guard Health Affairs Institutional Review Board, Riyadh, Saudi Arabia (Study Number: RC20/012/R). Participants' confidentiality was strictly observed throughout the study using the anonymous unique serial number for each subject and restricting data only to the investigators. Informed consent was not required due to the research's method as per the governmental and local research center's policy.

\section{Availability of data and material}

The datasets used and/or analyzed during the current study are available from the corresponding author on reasonable request.

\section{Consent for publication}

Not applicable.

\section{Competing interests}

No author has a conflict of interest in this study.

\section{References}

1. Hobbs A, Shea K, Roberts K, Daley M. Implications of Augmented Renal Clearance on Drug Dosing in Critically III Patients: A Focus on Antibiotics. Pharmacotherapy: The Journal of Human Pharmacology and Drug Therapy. 2015;35(11):1063-1075.

2. Fuster-Lluch O, Gerónimo-Pardo M, Peyró-García R, Lizán-García M. Glomerular Hyperfiltration and Albuminuria in Critically ILL Patients. Anaesthesia and Intensive Care. 2008;36(5):674-680. 
3. Sunder-Plassmann G, Hörl W. A critical appraisal for definition of hyperfiltration. American Journal of Kidney Diseases. 2004;43(2):396.

4. Udy A, Roberts J, Shorr A, Boots R, Lipman J. Augmented renal clearance in septic and traumatized patients with normal plasma creatinine concentrations: identifying at-risk patients. Critical Care. 2013;17(1):R35.

5. Kawano Y, Morimoto S, Izutani Y, Muranishi K, Kaneyama H, Hoshino K et al. Augmented renal clearance in Japanese intensive care unit patients: a prospective study. Journal of Intensive Care. 2016;4(1).

6. Garrelts J, Peterie J. Altered vancomycin dose vs. serum concentration relationship in burn patients. Clinical Pharmacology and Therapeutics. 1988;44(1):9-13.

7. Brater D, Bawdon R, Anderson S, Purdue G, Hunt J. Vancomycin elimination in patients with burn injury. Clinical Pharmacology and Therapeutics. 1986;39(6):631-634.

8. Loirat P, Rohan J, Baillet A, Beaufils F, David R, Chapman A. Increased Glomerular Filtration Rate in Patients with Major Burns and Its Effect on the Pharmacokinetics of Tobramycin. New England Journal of Medicine. 1978;299(17):915-919.

9. Bilbao-Meseguer I, Rodríguez-Gascón A, Barrasa H, Isla A, Solinís M. Augmented Renal Clearance in Critically III Patients: A Systematic Review. Clinical Pharmacokinetics. 2018;57(9):1107-1121.

10. Mahmoud S, Shen C. Augmented Renal Clearance in Critical Illness: An Important Consideration in Drug Dosing. Pharmaceutics. 2017;9(4):36.

11. Tröger U, Drust A, Martens-Lobenhoffer J, Tanev I, Braun-Dullaeus R, Bode-Böger S. Decreased meropenem levels in Intensive Care Unit patients with augmented renal clearance: benefit of therapeutic drug monitoring. International Journal of Antimicrobial Agents. 2012;40(4):370-372.

12. Carlier M, Carrette S, Roberts J, Stove V, Verstraete A, Hoste E et al. Meropenem and piperacillin/tazobactam prescribing in critically ill patients: does augmented renal clearance affect pharmacokinetic/pharmacodynamic target attainment when extended infusions are used?. Critical Care. 2013;17(3):R84.

13. Huttner A, Von Dach E, Renzoni A, Huttner B, Affaticati M, Pagani L et al. Augmented renal clearance, low $\beta$-lactam concentrations and clinical outcomes in the critically ill: An observational prospective cohort study. International Journal of Antimicrobial Agents. 2015;45(4):385-392.

14. Arabi Y, Alshimemeri A, Taher S. Weekend and weeknight admissions have the same outcome of weekday admissions to an intensive care unit with onsite \intensivist coverage*. Critical Care Medicine. 2006;34(3):605-611.

15. Aleidan F, Alkhelaifi $H$, Alsenaid A, Alromaizan $H$, Alsalham F, Almutairi A et al. Incidence and risk factors of carbapenem-resistant Enterobacteriaceae infection in intensive care units: a matched case-control study. Expert Review of Anti-infective Therapy. 2020;19(3):393-398.

16. "The Clinical and Laboratory Standards Institute (CLSI) M60 Performance Standards for Antifungal Susceptibility Testing of Yeasts, 2nd Edition 2020 edition," 2020, Sep. 02, 2020. ." 
17. Balaban, N. Q., Helaine, S., Lewis, K., Ackermann, M., Aldridge, B., Andersson, D. I., ... \& Zinkernagel, A. (2019). Definitions and guidelines for research on antibiotic persistence. Nature Reviews Microbiology, 17(7), 441-448.

18. Claus B, Hoste E, Colpaert K, Robays H, Decruyenaere J, De Waele J. Augmented renal clearance is a common finding with worse clinical outcome in critically ill patients receiving antimicrobial therapy. Journal of Critical Care. 2013;28(5):695-700.

19. Carrié C, Petit L, d'Houdain N, Sauvage N, Cottenceau V, Lafitte M, Foumenteze C, Hisz Q, Menu D, Legeron R, Breilh D, Sztark F. Association between augmented renal clearance, antibiotic exposure and clinical outcome in critically ill septic patients receiving high doses of $\beta$-lactams administered by continuous infusion: a prospective observational study. Int J Antimicrob Agents. 2018 Mar;51(3):443449. doi: 10.1016/j.jjantimicag.2017.11.013. Epub 2017 Nov 24. PMID: 29180280. F

20. Dulhunty JM, Roberts JA, Davis JS, Webb SA, Bellomo R, Gomersall C, et al. A multicenter randomized trial of continuous versus intermittent $\beta$-lactam infusion in severe sepsis. Am J Respir Crit Care Med 2015;192:1298-305.

21. Udy AA, Dulhunty JM, Roberts JA, Davis JS, Webb SAR, Bellomo R, Gomersall C, Shirwadkar C, Eastwood GM, Myburgh J, Paterson DL, Starr T, Paul SK, Lipman J; BLING-II Investigators; ANZICS Clinical Trials Group. Association between augmented renal clearance and clinical outcomes in patients receiving $\beta$-lactam antibiotic therapy by continuous or intermittent infusion: a nested cohort study of the BLING-II randomised, placebo-controlled, clinical trial. Int J Antimicrob Agents. 2017 May;49(5):624-630. doi: 10.1016/j.ijantimicag.2016.12.022. Epub 2017 Mar 9. PMID: 28286115.

\section{Tables}

\section{Table 1}


Augmented Renal Function

\begin{tabular}{|c|c|c|c|c|}
\hline Infections, n(\%) & Overall (133) & $\begin{array}{l}\text { Yes } \\
(\mathrm{N}=66)\end{array}$ & $\begin{array}{l}\text { No } \\
(\mathrm{N}=67)\end{array}$ & P-value \\
\hline Bacteremia & $20(15.0)$ & $8(12.1)$ & $12(17.9)$ & \multirow[t]{3}{*}{$0.64^{\wedge \wedge}$} \\
\hline Pneumonia & $91(68.4)$ & $47(71.2)$ & $44(65.7)$ & \\
\hline UTI & $22(16.5)$ & $11(16.7)$ & $11(16.4)$ & \\
\hline \multicolumn{5}{|l|}{ Origin of the Pathogen, $n(\%)$} \\
\hline Blood & $23(17.3)$ & $9(13.6)$ & $14(20.9)$ & \multirow[t]{3}{*}{$0.53^{\wedge \wedge}$} \\
\hline Respiratory & $88(66.2)$ & $46(69.7)$ & $42(62.7)$ & \\
\hline Urine & $22(16.5)$ & $11(16.7)$ & $11(16.4)$ & \\
\hline \multicolumn{5}{|l|}{ Organism, n(\%) } \\
\hline Klebsiella Pneumoniae & $42(31.6)$ & $22(33.3)$ & $20(29.9)$ & \multirow[t]{6}{*}{$0.70 * \star$} \\
\hline Pseudomonas Aeruginosa & $24(18.0)$ & $12(18.2)$ & $12(17.9)$ & \\
\hline Escherichia Coli (E. Coli) & $14(10.5)$ & $6(9.1)$ & $8(11.9)$ & \\
\hline Acinetobacter Spp & $11(8.3)$ & $7(10.6)$ & $4(6.0)$ & \\
\hline Streptococcus & $7(5.3)$ & $3(4.5)$ & $4(6.0)$ & \\
\hline Others & $30(23.2)$ & $12(18.1)$ & $18(27)$ & \\
\hline \multicolumn{5}{|l|}{ Antibiotics, n(\%) } \\
\hline Imipenem & $13(9.8)$ & $5(7.6)$ & $8(11.9)$ & \multirow[t]{3}{*}{$0.61^{\wedge \wedge}$} \\
\hline Meropenem & $52(39.1)$ & $25(37.9)$ & $27(40.3)$ & \\
\hline Piperacillin/Tazobactam & $68(51.1)$ & $36(54.5)$ & $32(47.8)$ & \\
\hline
\end{tabular}

-Denominator of the percentage is the total number of patients

${ }^{*} \mathrm{~T}$-Test / ^ Wilcoxon rank sum test is used to calculate the P-value.『

${ }^{\wedge}$ Chi-square test is used to calculate the P-value. $\mathbb{X}$

**Fisher Exact test is used to calculate the P-value.

\section{Table 2}

Regression analysis for the outcomes 


\section{Outcomes}

Augmented Renal Function

$\begin{array}{lllll}\text { Yes ARC } & \text { No ARC } & \begin{array}{l}\mathrm{P} \\ \text { value }\end{array} & \begin{array}{l}\text { Odds Ratio (OR) } \\ (95 \% \mathrm{Cl})\end{array} & \begin{array}{l}\mathrm{P} \text { - } \\ \text { value }\end{array} \\ (\mathrm{N}=66) & (\mathrm{N}=67) & & & \end{array}$

\section{Primary outcome}

$\begin{array}{llllll}\text { Eradication of pathogen at } & 48 / 66 & 48 / 67 & 0.09^{\wedge \wedge} & 1.08(0.41,2.78) & 0.88^{\star \wedge} \\ 10-14 \text { days, } \mathbf{n}(\%) & (72.7 \%) & (71.6 \%) & & & \end{array}$

\section{Secondary outcomes}

\begin{tabular}{llllll}
$\begin{array}{l}\text { Resistance after } 3 \text { days, } \mathbf{n} \\
(\%)\end{array}$ & $\begin{array}{l}12 / 66 \\
(18.2 \%)\end{array}$ & $\begin{array}{l}11 / 67 \\
(16.4 \%)\end{array}$ & $0.79^{\wedge \wedge}$ & $0.78(0.25,2.40)$ & 0.66 \\
\hline
\end{tabular}

\section{Pattern of resistance for} culture, $n$ (\%)

\begin{tabular}{|c|c|c|c|c|c|}
\hline Sensitive & $\begin{array}{l}51 / 66 \\
(77.3 \%)\end{array}$ & $\begin{array}{l}47 / 67 \\
(70.1 \%)\end{array}$ & $0.56^{* \star}$ & & \\
\hline MDR & $\begin{array}{l}10 / 66 \\
(15.2 \%)\end{array}$ & $\begin{array}{l}14 / 67 \\
(20.9 \%)\end{array}$ & & & \\
\hline XDR & $\begin{array}{l}4 / 66 \\
(6.1 \%)\end{array}$ & $6 / 67(9.0 \%)$ & & & \\
\hline PDR & $\begin{array}{l}1 / 66(1.5 \\
\%)\end{array}$ & $0 / 67(0)$ & & & \\
\hline $\begin{array}{l}\text { Persistence after } 3 \text { days, } n \\
\text { (\%) }\end{array}$ & $\begin{array}{l}21 / 66 \\
(31.8 \%)\end{array}$ & $\begin{array}{l}21 / 67 \\
(31.3 \%)\end{array}$ & $0.95^{\wedge \wedge}$ & $0.88(0.35,2.18)$ & $0.78^{\star \wedge}$ \\
\hline \multirow[t]{2}{*}{ 30-day ICU mortality, n (\%) } & $\begin{array}{l}3 / 66 \\
(4.5 \%)\end{array}$ & $\begin{array}{l}9 / 67 \\
(13.4 \%)\end{array}$ & $0.07^{\wedge \wedge}$ & $0.22(0.04,1.4)$ & $0.11^{\star \wedge}$ \\
\hline & & & & $\begin{array}{l}\text { beta coefficient } \\
\text { (Estimates) } \\
(95 \% \mathrm{Cl})\end{array}$ & $\begin{array}{l}\mathrm{P} \text { - } \\
\text { value }\end{array}$ \\
\hline $\begin{array}{l}\text { Hospital length of stay } \\
\text { (days), Median (Q1, Q3) \& }\end{array}$ & $\begin{array}{l}51.0 \\
(35.00 \\
120.00)\end{array}$ & $\begin{array}{l}30.0(23.00 \\
71.50)\end{array}$ & $0.07^{\wedge}$ & $0.2(-0.29,0.75)$ & $0.38^{\wedge \wedge}$ \\
\hline $\begin{array}{l}\text { ICU Length of Stay (days), } \\
\text { Median (Q1, Q3) \& }\end{array}$ & $\begin{array}{l}22.0 \\
(12.00, \\
34.00)\end{array}$ & $\begin{array}{l}17.50 \\
(13.00,26.00)\end{array}$ & $0.37^{\wedge}$ & $0.201(-0.18,0.58)$ & $0.30^{\wedge \wedge}$ \\
\hline
\end{tabular}




\section{Outcomes}

Augmented Renal Function

$\begin{array}{lllll}\text { Yes ARC } & \text { No ARC } & \begin{array}{l}\mathrm{P} \\ \text { value }\end{array} & \begin{array}{l}\text { Odds Ratio (OR) } \\ (95 \% \mathrm{Cl})\end{array} & \begin{array}{l}\mathrm{P} \text { - } \\ \text { value }\end{array} \\ (\mathrm{N}=66) & (\mathrm{N}=67) & & & \end{array}$

-Denominator of the percentage is the total number of patients

${ }^{*} \mathrm{~T}$-Test / ${ }^{\wedge}$ Wilcoxon rank sum test is used to calculate the P-value.

${ }^{\wedge}$ Chi-square test is used to calculate the P-value.

**Fisher Exact test is used to calculate the P-value.

${ }^{* \wedge}$ Multivariate Logistic regression is used after adjusting for Gender and mean weight used depending on BMI to calculate Odds ratio and p-value

${ }^{\wedge} \wedge$ Multivariate Negative binomial regression is used after adjusting for Gender and mean weight used depending on BMI to calculate estimates and p-value

\section{Supplementary Files}

This is a list of supplementary files associated with this preprint. Click to download.

- Supplementary1.docx

- Supplementary2.docx

- FlowChartARC.docx 